\title{
MULTIPOLAR INTRAFASCICULAR STIMULATION
}

\author{
Jan H. Meier and Wim L.C. Rutten, Member, IEEE \\ Biomedical Engineering Division, Department of Electrical Engineering \\ Twente University, P.O. Box 217, 7500 AE Enschede, the Netherlands
}

\begin{abstract}
The suppressing effect of two intrafascicular anodes on the neural recruitment elicited by one intrafascicular cathode has been studied. Recruitment curves are calculated with a nerve stimulation model and are compared to experimental curves for the peroneal nerve of rat.
\end{abstract}

\section{INTRODUCTION}

Theoretically the stimulating effect of an intrafascicular monopolar cathode can be locally suppressed by the presence of additional anodes. This enables the creation of restricted excitation regions with a higher spatial degree of selectivity [1].

In this paper the effect of the anodes will be studied quantitatively. Recruitment curves will be simulated with a nerve stimulation model and will be compared to experimental curves as measured in rat.

\section{THEORY}

For stimulation with a short stimulus pulse the rise of the transmembrane potential $\Delta V$ at a node of Ranvier with position $(x, y, z)$ is proportional to the pulse duration $T$ and the activating function $f(x, y, z, \lambda)[1]$.

$$
\Delta \mathrm{V}(\mathrm{x}, \mathrm{y}, \mathrm{z})=\mathrm{KTf}(\mathrm{x}, \mathrm{y}, \mathrm{z}, \lambda)
$$

$\lambda$ is the internodal length of the corresponding nerve fibre. $\mathrm{K}$ is a constant. The node/fibre is activated when $\Delta \mathrm{V}$ surpasses a threshold value $V_{\text {thresh }}$. For a cylindrical fascicle along the $\mathrm{z}$-axis with radius $\mathrm{r}$, the activation probability $\mathrm{F}$ of any fibre is

$$
F(T, \lambda)=\left(\pi r^{2} \lambda\right)^{-1} \underset{-r}{\int} \int_{-\sqrt{r^{2}-x^{2}}}^{r} \int_{0}^{\sqrt{r^{2}-x^{2}}} \lambda \operatorname{Himax}\{\ldots,
$$

$\left.f(x, y, z-\lambda, \lambda), f(x, y, z, \lambda), .\}.-V_{\text {thresh }} K^{-1} T^{-1}\right] d z d y d x$

The function $\max \{. . .$.$\} returns the maximum of all argu-$ ments, $H[u]$ is the Heaviside unit (step) function of $u$. The probability function $\mathrm{F}$ can be interpreted as a recruitment curve for nerve fibres with a given internodal length $\lambda$. A change in $\mathrm{T}$ has the same effect as a change of the stimulus current I; $\mathrm{F}(\mathrm{T}, \lambda) \sim \mathrm{F}(\mathrm{I}, \lambda)$.

0-7803-0785-2/92\$03.00 @IEEE

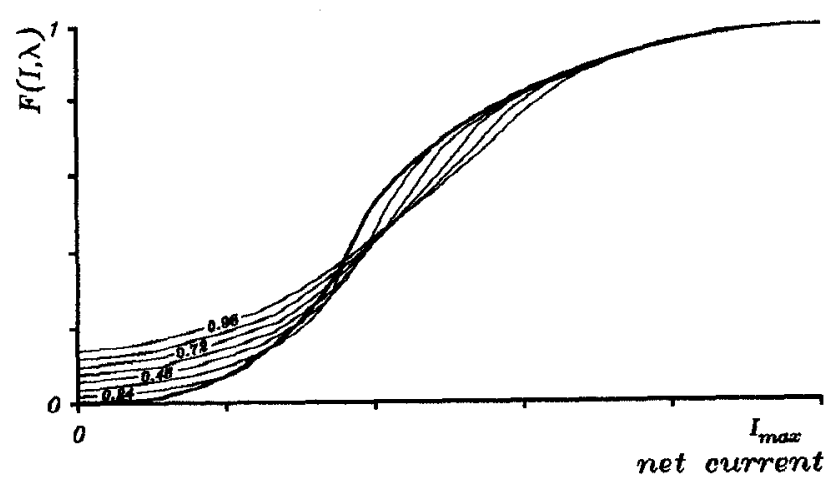

Fig. 1 The recruitment probability with an tripole versus the net current.

Fig. 1 shows the recruitment probability versus the algebraic net current of the electrodes for an electrode configuration consisting of a cathode, flanked by two anodes on opposite sites of the cathode. The axis of this tripole is transverse to the nerve fibres. The interelectrode distance is $150 \mu \mathrm{m}$ and the internodal length is set to $\lambda=1000 \mu \mathrm{m}$. The dimensions and conductivities of the cylindrical nerve bundle have been given values as to mimic the peroneal nerve of rat [1].

For each curve the cathodal current is kept constant (at a value given by the indices as a fraction of $I_{\max }$, the current value at which all the fibres are being recruited by cathodal monopolar stimulation). For comparison also the (thick) monopolar curve is shown.

For small anodal currents (large net current) the recruitment probability for the tripole is lower than for pure monopolar stimulation with the same net current. If the anodal currents are increased the recruitment probability becomes higher than that of the monopole.

\section{EXPERIMENTS}

A stimulation device [2] ccntaining an array of electrode sites at an interelectrode distance of $50 \mu \mathrm{m}$ was pierced into the peroneal nerve of rat. The summed twitch contraction forces of the $\mathrm{E}$ (xtensor) $\mathrm{D}$ (igitorum) $\mathrm{L}$ (ongus) and the Tibial muscles, elicited by rectangular $100 \mu \mathrm{s}$ stimulus pulses, were measured isometrically. 


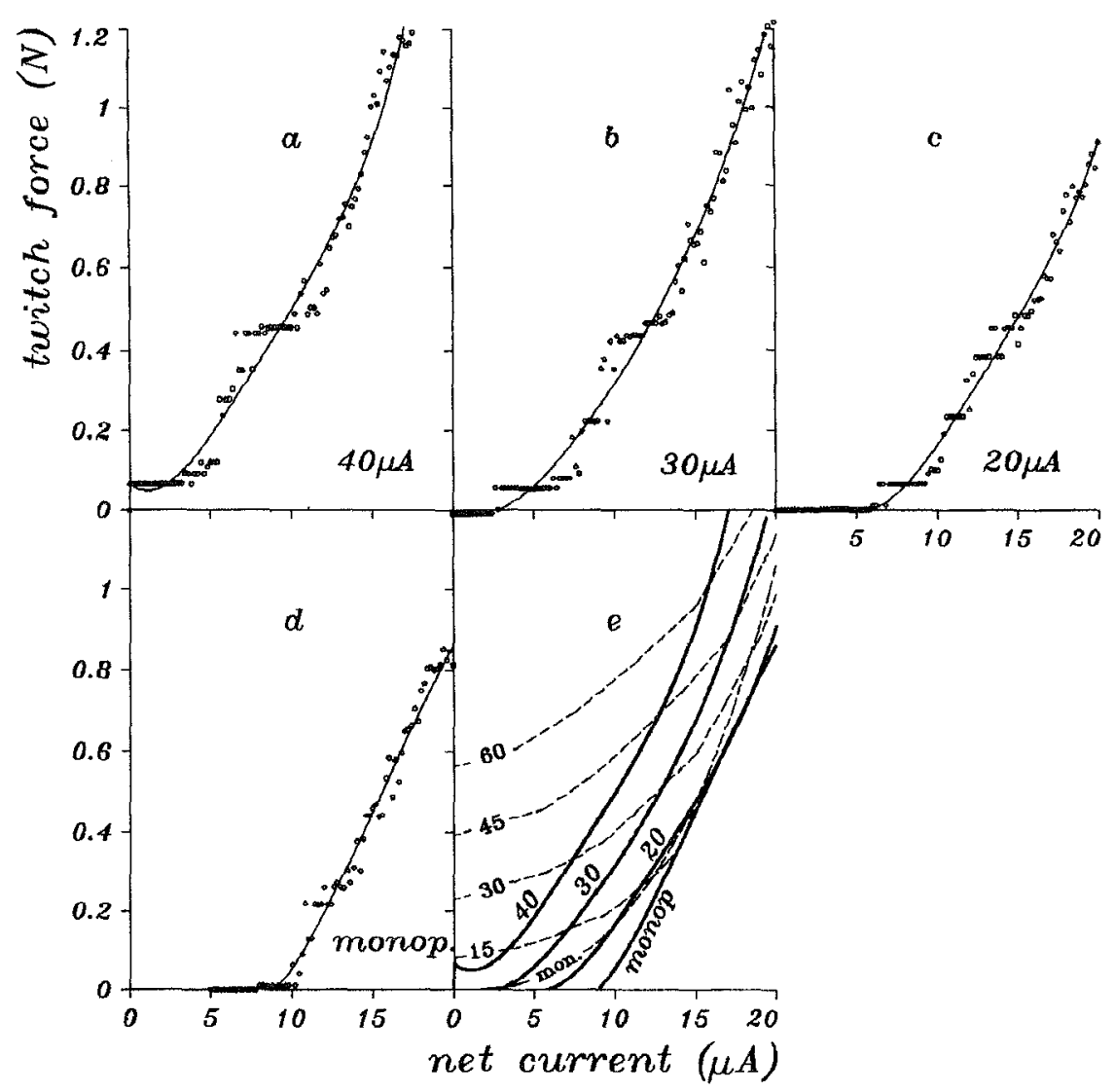

Fig. 2 Recruitment data for tripolar stimulation with cathodal currents of 40,30 and $20 \mu \mathrm{A}$ as well as monopolar recruitment data (4d).

As an example in fig. 2 recruitment data for tripolar stimulation with an interelectrode distance of $150 \mu \mathrm{m}$ are shown as well as for monopolar stimulation. Discrete levels of twitch force can be seen as a result of the activation of a discrete number of motorunits. Each measurement series was fitted to a 4-th degree polynomial.

These polynomials are also shown in fig. $2 \mathrm{e}$ in which they are compared to the theoretical curves from fig. 1 with $\mathrm{I}_{\max }$ $=62.5 \mu \mathrm{A}$ and $\mathrm{F}_{\max }=4 \mathrm{~N}$. Both theoretically and experimentally the curve with the lowest cathodal current is closest to the monopolar recruitment curve. An increasing cathodal current results in higher recruitment levels.

Quantitatively the theoretical and experimental curves do not compare well. It is to be noted that the experimental recruitment curves vary strongly with the position of the stimulation device (not shown in this paper) which makes any individual comparison casuistic.

\section{CONCLUSIONS}

Quantitatively the experimental recruitment curves are well explained by the model used. Cathodal stimulation effects can be locally suppressed by the presence of anodes.

At low stimulation levels the experimental recruitment curves are strongly dependent on the position of the electrode configuration which prevents a direct comparison of individual experiments with the (average) calculations. Further analysis of the various recruitment curves (not shown in this paper) suggests that the motor fibres which innervate the same muscle are grouped.

\section{REFERENCES}

[1] J.H. Meier, W.L.C. Rutten, A.E. Zoutman, H.B.K. Boom, and P. Bergveld, "Simulation of multipolar fiber selective neural stimulation using intrafascicular electrodes," IEEE Trans. Biomed. Eng., vol. 39/2, pp. 122-134, 1992.

[2] W.L.C. Rutten, H.J. van Wier, and J.H.M. Put, "Sensitivity and selectivity of intraneural stimulation using an intraneural multi electrode stimulation device in silicon technology," IEEE Trans. Biomed. Eng., vol. 38/2, pp. 192-198, 1991. 